\title{
Calorimetric Study on Plastic-to-Rubber Transition in a Styrene-Butadiene-Styrene Triblock Copolymer
}

\author{
Keiichiro ADACHI, Shoichi FUKUMURA, and Tadao KotAKA \\ Department of Macromolecular Science, Faculty of Science, \\ Osaka University, Toyonaka, Osaka 560, Japan
}

(Received February 20, 1984)

\begin{abstract}
Heat evolution from and mechanical work done on films of a styrene-butadienestyrene (SBS) triblock copolymer were investigated under tensile strain. The relationship between microphase structure and thermomechanical behavior was examined on SBS films having lamellar, cylindrical, and spherical morphology which were cast from solutions in three mixed solvents. For SBS films having lamellar and cylindrical morphology, the enthalpy changes due to the plastic-torubber transition were found to be 0.24 and $0.17 \mathrm{~J} \mathrm{~cm}^{-3}$, respectively. These values correspond to the enthalpy required for irreversible destruction of the polystyrene domains. On the other hand, an SBS film having spherical polystyrene domains exhibited behavior similar to that of a vulcanized rubber.
\end{abstract}

KEY WORDS Styrene-Butadiene-Styrene Triblock Copolymer / Heat of

Deformation / Thermoplastic Elastomer / Enthalpy of Deformation /

Microphase Structure / Plastic-to-Rubber Transition / Calorimetry /

Mechanical properties of thermoplastic elastomers such as styrene-butadiene-styrene (SBS) triblock copolymers have been investigated by many authors, and the relationship between their mechanical properties and microphase structure has been well established. ${ }^{1,2}$ An SBS film with spherical polystyrene domains dispersed in the polybutadiene matrix exhibits rubber-like behavior, since the hard polystyrene domains work as crosslinks. In contrast, an SBS film having lamellar morphology shows properties similar to plastics, i.e., its stress-strain curve shows an initial steep rise, followed by a yielding and cold-drawing region. Following this yielding region, the SBS film is transformed irreversibly to a rubber-like soft material, presumably because the continuous polystyrene domains have been destroyed during the initial stretching period. This phenomenon is referred to as straininduced plastic-to-rubber transition. ${ }^{2}$

The changes in mechanical property caused by the plastic-to-rubber transition and the corresponding change in the microphase structure have been investigated by several authors. $^{3-10}$ For an SBS sample and its blends with polystyrene and with polybutadiene, the structural change due to the transition was studied by electron microscopy (EM). ${ }^{13)}$ Kawai et $a l^{2,4,5}$ studied the breakdown of the microphase structure of SBS samples by EM and small angle X-ray scattering (SAXS). It was found that the glassy polystyrene phase exhibits considerable plastic flow before the breakdown. A similar study was reported by Aggarwal $^{9}$ for high impact polystyrenes. However, few calorimetric studies have been made on the change in microphase structure due to the plastic-to-rubber transition except measurements of the glass transition temperature by differencial scanning calorimetry (DSC). ${ }^{8}$ In this paper, we report the changes in thermodynamic properties of SBS films accompanying the plastic-to-rubber transition caused by tensile deformation.

Regardless of thermodynamic reversibility, 
the enthalpy change $\Delta H$ in a system is given by

$$
\Delta H=W+Q
$$

where $W$ and $Q$ denote the work done on and the heat absorbed by the system, respectively. In this study, $W$ and $-Q$ associated with the deformation of SBS films were measured simultaneously and $\Delta H$ was calculated from eq 1 .

Several authors ${ }^{11-15}$ studied thermomechanical behavior of vulcanized rubbers. In their studies, the data were analyzed assuming the change caused by deformation to be quasistatic and reversible. In the present systems, irreversible structural changes due to the plastic-to-rubber transition should play an important role. Therefore, the thermomechanical behavior of SBS films should be quite sensitive to morphology, and hence should differ from that of vulcanized rubbers. In order to clarify the influence of morphology on the thermomechanical properties, $W$ and $-Q$ were measured on SBS films with different morphology cast from different solvents. ${ }^{3}$

\section{EXPERIMENTAL}

\section{Materials}

A commercial SBS containing 33\% styrene (Shell Chemical Co., TR-1101) was used. It was reported ${ }^{3}$ that the number-average molecular weights of its styrene and butadiene blocks were $1.57 \times 10^{4}$ and $6.37 \times 10^{4}$, respectively and its content of 1,4-cis, 1,4-trans, and 1,2-vinyl linkages in the polybutadiene block was 37,55 , and $8 \%$, respectively. Films of about $1 \mathrm{~mm}$ thick were prepared by casting $5 \%$ solutions of the sample dissolved in the following three mixed solvents: tetrahydrofuran/ methylethyl ketone (volume ratio $\mathrm{T} / \mathrm{M}=9 / 1$ ), cyclohexane/benzene $(\mathrm{C} / \mathrm{B}=1 / 1), \quad$ and cyclohexane $/ n$-hexane $(C / N=3 / 1)$. One week after the casting, the films were dried in vacuo of about $10^{-1} \mathrm{~Pa}$ at room temperature for several days. They were coded as SBS (T/M), SBS $(\mathrm{C} / \mathrm{B})$, and SBS $(\mathrm{C} / \mathrm{N})$ according to the casting solvents. The films were cut into pieces of $25 \times 3 \times 1 \mathrm{~mm}^{3}$ by a sharp knife and used for calorimetric measurements.

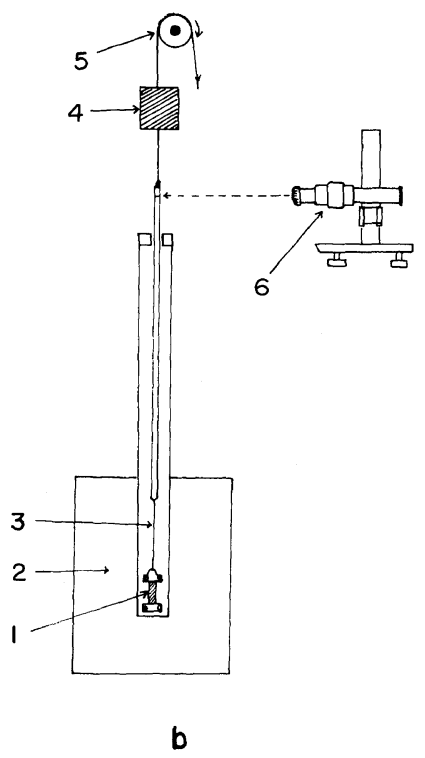

Figure 1. a) Sample holder for the measurement of heat of deformation. 1, sample; 2, clamp; 3, string (steel wire).

b) Schematic view of the calorimeter. 1, sample; 2 , microcalorimeter; 3 , string; 4, strain gauge; 5 , motor; 6 , cathetometer. 


\section{Methods}

The heat of deformation was measured at $313 \mathrm{~K}$ with a microcalorimeter (Tokyo Riko, Model SCC-1) described previously. ${ }^{16}$ Figure 1a shows the sample holder used, and Figure $1 \mathrm{~b}$ a schematic view of the instrument. The specimen between the clamps was about $10 \mathrm{~mm}$ long. The clamped specimen was stretched at a rate of $0.83 \mathrm{~mm} \mathrm{~min}^{-1}$ until the tensile strain reached about 0.2 . The heat evolved during this operation was converted into an electric signal and traced on a pen recorder until it decreased to a detectable lowest limit ( $c a \cdot 10^{-5} \mathrm{~J} \mathrm{~s}^{-1}$ ). At the same time, the change in the sample length and the tensile force were measured by a cathetometer and a stress gauge, respectively. It took about $20 \mathrm{~min}$ to complete the procedure. The same procedure was repeated until the total extention ratio $\lambda$ reached about 2.5 . The work $W$ was determined by numerical integration of the tensile stress-strain curve.

\section{RESULTS}

The dependence of $W$ and $-Q$ on $\lambda$ for SBS $(\mathrm{T} / \mathrm{M})$, SBS $(\mathrm{C} / \mathrm{B})$, and SBS $(\mathrm{C} / \mathrm{N})$ is shown in Figures 2, 3, and 4, respectively. These figures are for the as-cast films (1st cycle) in both stretching and releasing directions and for the films stretched once ( 2 nd cycle). The arrows indicate the directions of deformation. In the stretching part of the 1st cycle for SBS (T/M) (see Figure 2), the value of $W$ is greater than that of $-Q$, differing from vulcanized rubbers. On the other hand, the magnitudes of $W$ and $-Q$ for $\mathrm{SBS}(\mathrm{C} / \mathrm{N})$ are about the same, indicating that entropic elasticity is dominant as in vulcanized rubbers. It is seen that SBS (C/B) exhibits behavior intermediate between SBS $(\mathrm{T} / \mathrm{M})$ and SBS $(\mathrm{C} / \mathrm{N})$. On the other hand, the behavior of the three samples in the 2 nd cycle are similar, indicating that the features observed in the 1st cycle were lost in the deformation part of the 1st cycle. Obviously, this is due to a plastic-to-rubber transition.

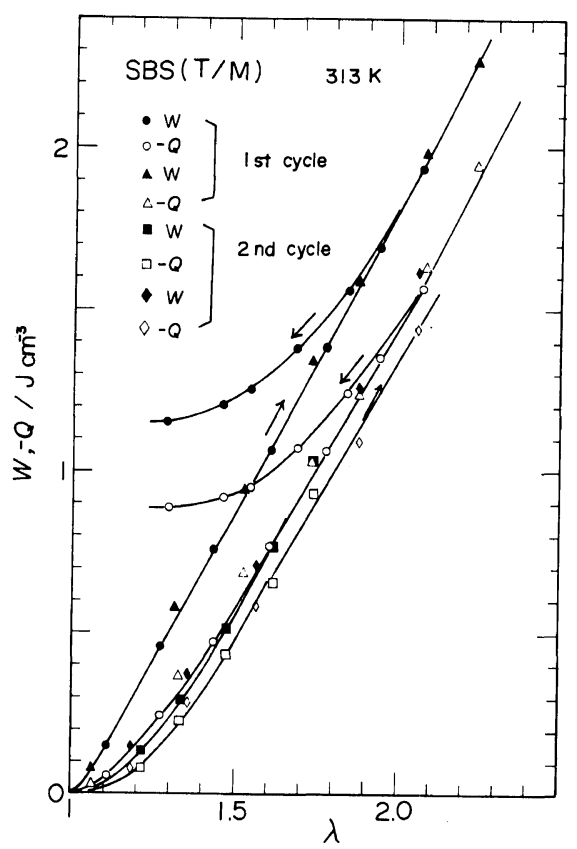

Figure 2. Dependence of $W$ and $-Q$ on extension ratio $i$ for SBS $(T / M)$ at $313 \mathrm{~K}$. Data represented by 0 , $\bigcirc, \boldsymbol{\square}$, and $\square$ were obtained at a rate of extension of $0.83 \mathrm{~mm} \mathrm{~min}^{-1}$, while $\boldsymbol{\Delta}, \Delta, \diamond$, and $\diamond$ at 10 $\mathrm{mm} \mathrm{min}^{-1}$.

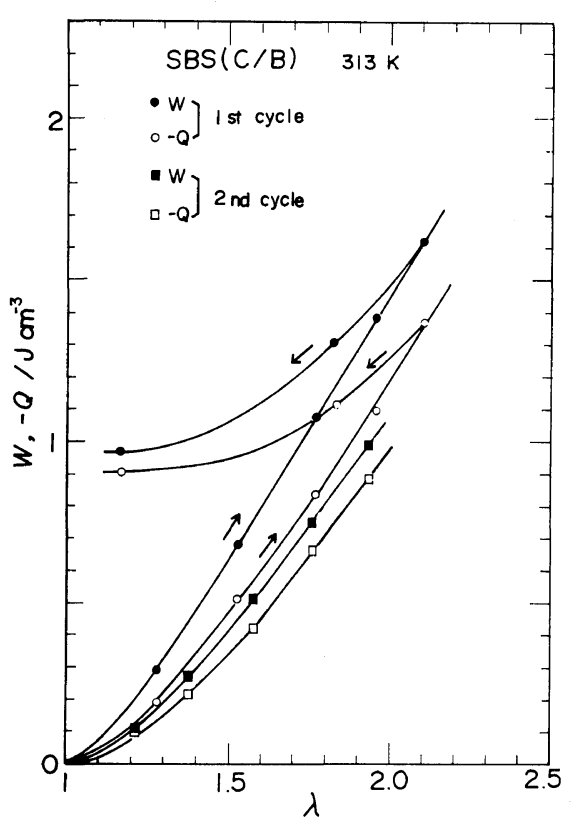

Figure 3. Dependence of $W$ and $-Q$ on extension ratio $\lambda$ for $\operatorname{SBS}(\mathrm{C} / \mathrm{B})$. 


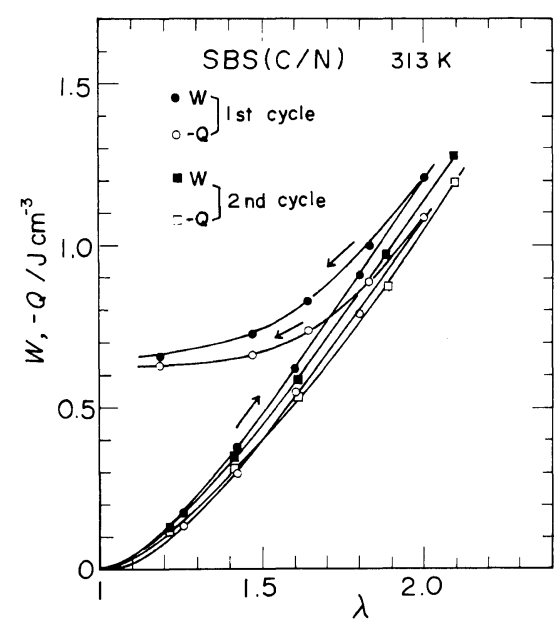

Figure 4. Dependence of $W$ and $-Q$ on extension ratio $i$ for $\operatorname{SBS}(\mathrm{C} / \mathrm{N})$.

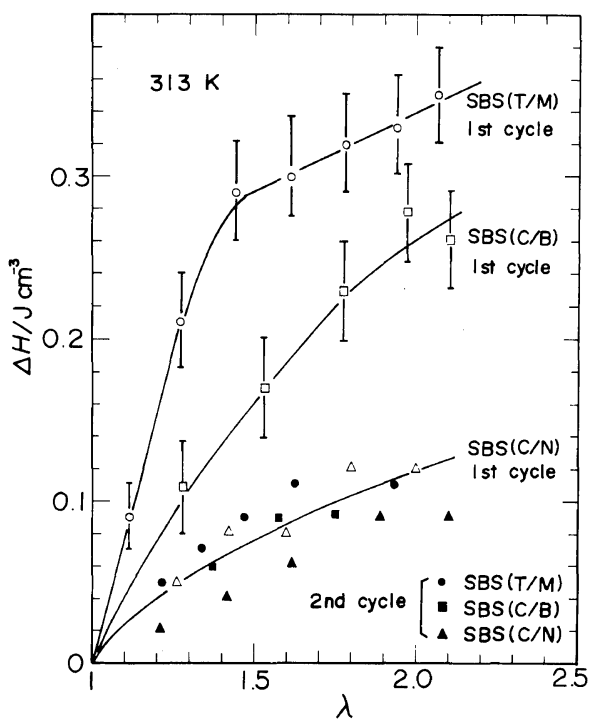

Figure 5. The change in enthalpy $\Delta H$ caused by tensile strain.

For all the three samples, stress relaxation was observed during the measurement of heat evolution under a constant strain. Creep was also observed after the 1st cycle, i.e., the length of SBS (T/M), SBS (C/B), and SBS (C/N) films in the stress free state increased by 16,22 , and $15 \%$ of the original length, respectively. The value of $\lambda$ for the releasing part of the 1 st cycle was calculated from the film length relative to the original length. However, before starting the 2nd cycle, the film length at the relaxed state was remeasured to correct for the effect of creep.

Figure 5 shows the enthalpy changes $\Delta H$ determined from these data and eq 1 . Effects of stretching rate on $W$ and $-Q$ were also examined with SBS $(\mathrm{T} / \mathrm{M})$. The results obtained at the rates of 0.83 and $10 \mathrm{~mm} \mathrm{~min}^{-1}$ are shown in Figure 2. It can be seen that the effect of stretching rate was negligible.

\section{DISCUSSION}

\section{Effect of Morphology on the Thermomechanical \\ Behavior}

It was reported that the polystyrene domains of SBS $(T / M)$ and SBS $(C / B)$ films are lamella and cylinder-like, respectively. ${ }^{3}$ Probably, SBS $(\mathrm{C} / \mathrm{N})$ has spherical polystyrene domains dispersed in the polybutadiene matrix, since $n$-hexane used for film casting is a nonsolvent for polystyrene. The fact that the tensile modulus of SBS $(\mathrm{C} / \mathrm{N})$ was smaller than that of SBS (C/B) also supports this consideration, since SBS films having a discontinuous polystyrene phase have an elastic modulus lower than those having a continuous polystyrene phase.

As shown in Figure 5, $\Delta H$ of SBS (T/M) rises steeply in the range of $\lambda$ between 1 and 1.4. Since yielding was observed around $\lambda=$ 1.3 , this rise in $\Delta H$ may be ascribed to the plastic-to-rubber transition. ${ }^{3}$ Thus, the measured $\Delta H$ should be the enthalpy required for breaking the polystyrene domains. ${ }^{1-10}$ For $\lambda$ below 1.3, it may be considered that the external tensile force is supported by the glassy polystyrene domain. Therefore, the stress should be energetic. In fact, $W$ for the SBS (T/M) film was 2 to 3 times larger than $-Q$ in the range of $\lambda<1.3$. However, $-Q$ in this range was not negligible. Therefore, the observed heat evolution in the range of small extension ratio must be attributed partly to the 
entropy change in the polybutadiene phase and partly to the irreversible heat production due to the destruction of the polystyrene domains.

On the other hand, $\Delta H$ of SBS (C/B) exhibited a gradual increase with increasing $\lambda$. Thus, the plastic-to-rubber transition or the stress-induced softening in SBS (C/B) occurred more gradually than that in SBS $(\mathrm{T} / \mathrm{M})$. This behavior is compatible with the stress-strain behavior of SBS (C/B), which did not exhibit yielding. ${ }^{3}$

It was found that SBS $(\mathrm{C} / \mathrm{N})$ gave smaller $W$ and $-Q$ than $\operatorname{SBS}(\mathrm{T} / \mathrm{M})$ and $\operatorname{SBS}(\mathrm{C} / \mathrm{B})$. The ratio of $W$ to $-Q$ was close to unity, indicating that the stress was entropic as in vulcanized rubbers. As shown in Figure 2-4, all the three samples exhibited irreversible $W$ and $-Q$ versus $\lambda$ curves, i.e., $W$ and $-Q$ in the contraction process did not agree with those in the extension process. This may be due partly to the plastic-to-rubber transition and partly to the creep of the sample.

\section{Thermomechanical Model}

The irreversible thermomechanical behavior was analyzed using a thermomechanical model shown in Figure 6; in this model, the elements 1 and 2 represent enthalpic and entropic springs, respectively. The enthalpic spring stores the work $\left(W_{\mathrm{H}}\right)$ done on it as a strain energy. Therefore, it absorbs or evolves no heat in stretching and contraction. On the other hand, the work $\left(W_{\mathrm{s}}\right)$ done in stretching

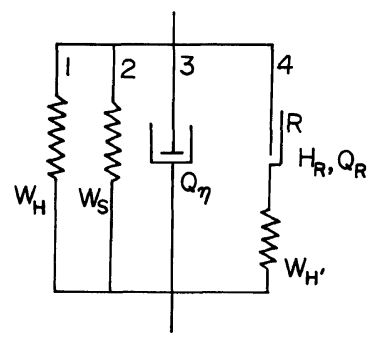

Figure 6. Mechanical model which represents the thermomechanical behavior of the SBS films. the spring 2 causes a decrease in entropy and vice versa. Thus, the entropy change $\Delta S$ evolves or absorbs heat equal to $T \Delta S$ $\left(=-W_{\mathrm{S}}\right)$. The element 3 is a dash-pot representing an irreversible energy dissipation due to internal friction. This element evolves heat $-Q_{\eta}$ which is proportional to the absolute value of $\mathrm{d} \lambda / \mathrm{d} t$, where $t$ denotes time. However, since the observed $W$ and $-Q$ were almost independent of the rate of stretching as shown in Figure 2, this element does not play a dominant role in the present system. Hence, we assume that $-Q_{\eta}=0$.

The element 4 consists of an enthalpic spring $\left(W_{\mathrm{H}^{\prime}}\right)$ and a ratchet or a slider (denoted $\mathrm{R}$ in Figure 6), which undergoes a plastic-to-rubber transition. When a stress greater than the yield strength of the ratchet is applied, it is disconnected and never reformed. We assume that when the ratchet is disconnected, part of enthalpy $W_{\mathbf{H}^{\prime}}$ is spent for disconnecting the ratchet and stored as a potential energy $\Delta H_{\mathrm{R}}$, but the rest is dissipated by friction in the process of disconnecting the ratchet to heat $-Q_{\mathrm{R}}$. Thus, $W$ and $-Q$ for the stretching part of the first cycle $\left(W_{1 \mathrm{st}}\right.$ and $\left.-Q_{1 \mathrm{st}}\right)$ in the state where the ratchet is disconnected are given by

$$
\begin{aligned}
& W \equiv W_{1 \mathrm{st}}=W_{\mathrm{H}}+W_{\mathrm{S}}+\Delta H_{\mathrm{R}}-Q_{\mathrm{R}} \\
& Q \equiv-Q_{1 \mathrm{st}}=W_{\mathrm{S}}-Q_{\mathrm{R}}
\end{aligned}
$$

Since the element 4 does not play a role in the 2nd cycle, $W$ and $-Q$ for the second cycle ( $W_{2 \text { nd }}$ and $-Q_{2 \text { nd }}$ ) may be given by

$$
\begin{aligned}
& W \equiv W_{2 \text { nd }}=W_{\mathrm{H}}+W_{\mathrm{S}} \\
& W \equiv-Q_{2 \text { nd }}=W_{\mathrm{S}}
\end{aligned}
$$

From the data of $W$ and $-Q$ for the SBS samples, we attempted to evaluate the parameters appearing in these equations at $\lambda=2.0$. In this calculation, it was necessary to take the effect of creep into account. Since the original length $l_{0}$ of the sample increased to $l_{0}{ }^{\prime}$ after the 1 st cycle, it was assumed that the values of $W_{\mathrm{H}}$ and $W_{\mathrm{S}}$ in the 1 st cycle at $\lambda=2.0$ were equal to 
those at $\lambda=2 l_{0} / l_{0}{ }^{\prime}$ in the $2 \mathrm{nd}$ cycle. The results of the calculation are summarized in Table I.

\section{Reversible Change}

The $W$ and $-Q$ versus $\lambda$ curves for SBS $(\mathrm{C} / \mathrm{N})$ in the 1st cycle and those for SBS $(\mathrm{T} / \mathrm{M})$ and SBS $(\mathrm{C} / \mathrm{B})$ in the 2 nd cycle were similar to those of vulcanized rubbers. Therefore, the thermomechanical properties of these samples may be explained in terms of the theory of rubber elasticity. In a system in which deformation goes reversibly, the tensile force $f$ at constant volume $V$ and temperature $T$ is given by

$$
f=(\partial A / \partial)_{V, T}=(\partial U / \partial l)_{V, T}-T\left(\partial S / \partial l_{V, T}\right.
$$

where $l$ denotes the length of the specimen, and $A, U$, and $S$ have the usual meaning. The first term in eq 6 is referred to as the energetic force $f_{\mathrm{e}}$. To determine the ratio $f_{\mathrm{e}} / f$ from thermomechanical data obtained under a constant pressure, Allen et al. ${ }^{13}$ used the relation

$$
f_{\mathrm{e}} / f=\left[\Delta H-V T \beta f^{\prime}\left(1-\alpha^{-1}\right) /\left(\alpha-\alpha^{-2}\right)\right] / W
$$

where $f^{\prime}$ is the force per unit cross-sectional area of the unstrained rubber, and $V, \alpha$, and $\beta$ denote the volume, the microscopic extension ratio in the polybutadiene phase and the thermal expansion coefficient, respectively. The value of $\alpha$ was estimated from the macroscopic extension ratio $\lambda$ by the Einstein-Guth-Gold equation $^{17}$

$$
\alpha=\left(1+2.5 c+14.1 c^{2}\right) \lambda
$$

where $c$ denotes the volume fraction of the filler. In the present system, $c$ refers to the polystyrene phase and is calculated to be 0.27 from the bulk densities of polystyrene and polybutadiene and the polystyrene content of the SBS sample. For polybutadiene ${ }^{18}$ having a microstructure similar to the polybutadiene block of the present SBS sample, $\beta$ is reported to be $7.5 \times 10^{-4} \mathrm{~K}^{-1}$.

With these data, $f_{\mathrm{e}} / f$ for $\mathrm{SBS}(\mathrm{C} / \mathrm{N})$ was calculated to be 0.091 and 0.068 at $\lambda=1.6$ and 2.0 , respectively. The $f_{\mathrm{e}} / f$ values for vulcanized polybutadiene were reported to be 0.05 to 0.17 by several authors. ${ }^{12,19-22}$ The present result is close to the data of Gent and Kuan, ${ }^{21}$ who reported $f_{\mathrm{e}} / f$ to be 0.05 to 0.09 . However, it should be noted that because of creep during the stretching process, the value of $\lambda$ included an error of about $15 \%$. The error in $f_{\mathrm{e}} / f$ due to creep was estimated to be 0.03 and 0.01 at $\lambda=1.6$ and 2.0 , respectively.

\section{Enthalpy of Plastic-to-Rubber Transition}

$\Delta H_{\mathrm{R}}$ in the mechanical model (Figure 6) was estimated to be 0.24 and $0.17 \mathrm{~J} \mathrm{~cm}^{-3}$ for SBS $(\mathrm{T} / \mathrm{M})$ and SBS $(\mathrm{C} / \mathrm{B})$, respectively. These values correspond to the enthalpies of the plasticto-rubber transition, and from the microscopic view point, they may be considered the energies for creating fracture surfaces in the polystyrene phase.

Using EM and SAXS, Hashimoto et al. ${ }^{2}$ studied the morphological change in the microdomain of a stretched SBS $(49 \%$ styrene content) specimen having lamellar morphology. They observed orientation, shearing, kinking and destruction of the polystyrene domains at $\lambda=1.8$, and also fragmentation of the disrupted domains when $\lambda$ was further increased to 6.0 . The electron micrograph reported previously ${ }^{3}$ for SBS (T/M) also appears to indicate that the polystyrene lamellae possess 10 to 50 crazes per $1 \mu \mathrm{m}$ at $\lambda=2.5$. Thus, the area $(s)$ of fracture surfaces created in the polystyrene domains as a result of the plasticto-rubber transition was estimated to range from $3 \times 10^{4}$ to $1.5 \times 10^{5} \mathrm{~cm}^{2}$ per unit volume of the sample.

Berry ${ }^{23}$ estimated the fracture surface energy $(\Gamma)$ of bulk polystyrene to be $0.17 \mathrm{~J} \mathrm{~cm}^{-2}$, using Griffith's theory. If this value is applied to the disrupted microdomains, the change in enthalpy due to the plastic-to-rubber transition $(=\Gamma s)$ ranges from $5 \times 10^{3}$ to $3 \times 10^{4}$ $\mathrm{J} \mathrm{cm}^{-3}$. These values are $10^{4}$ to $10^{5}$ times larger than the observed enthalpy of transition $\Delta H_{\mathrm{R}}$. 
Table I. The values of $W$ and $-Q$ at $\lambda=2.0$ in the 1 st cycle, those in the 2 nd cycle,$^{\mathrm{a}}$ and the parameters appearing in eq $2-5$ (unit: $\mathrm{J} \mathrm{cm}^{-3}$ )

\begin{tabular}{lccccccc}
\hline & $W_{1 \mathrm{st}}$ & $-Q_{1 \mathrm{st}}$ & $W_{\text {2nd }}$ & $-Q_{\text {2nd }}=W_{\mathrm{S}}$ & $W_{\mathrm{H}}$ & $\Delta H_{\mathrm{R}}$ & $-Q_{\mathrm{R}}$ \\
\hline SBS (T/M) & 1.80 & 1.43 & 0.96 & 0.86 & 0.10 & 0.24 & 0.57 \\
SBS (C/B) & 1.46 & 1.19 & 0.62 & 0.52 & 0.10 & 0.17 & 0.67 \\
SBS (C/N) & 1.21 & 1.08 & 0.78 & 0.68 & 0.10 & 0.03 & 0.40 \\
\hline
\end{tabular}

a The values of $W$ and $-Q$ in the 2 nd cycle for SBS $(\mathrm{T} / \mathrm{M}), \mathrm{SBS}(\mathrm{C} / \mathrm{B})$, and SBS $(\mathrm{C} / \mathrm{N})$ were those at $\lambda=1.72,1.64$, and 1.74 , respectively.

Thus, it can be concluded that the breakdown of styrene domains due to the plastic-to-rubber transition follow a mechanisms very different from the fracture mechanism for bulk polystyrene.

A probable reason for the discrepancy between $\Gamma s$ and $\Delta H_{\mathrm{R}}$ is as follows. When glassy polystyrene is fractured, scission of the main chains may occur, giving a high fracture surface energy. On the other hand, the fracture of polystyrene domains in SBS proceeds through a plastic flow of the domains. ${ }^{2,4,5}$ This implies that polystyrene chains are somewhat mobile, and therefore, they slip out of entanglements without scission of the chains. Thus, the fracture energy for the polystyrene domains in SBS $(\mathrm{T} / \mathrm{M})$ and SBS $(\mathrm{C} / \mathrm{B})$ should be much smaller than that for bulk polystyrene. It should be noted that the plastic flow of the polystyrene domains dissipates only the energy by friction $\left(Q_{\mathrm{R}}\right.$ in Table I). Hence, the flow does not contribute to the change in enthalpy.

We next examine whether the fracture surface energy of the polystyrene domain is equal to the interfacial free energy (interfacial tension $\gamma_{i}$ ) for the boundary between the polystyrene and polybutadiene phases. Since no experimental data of $\gamma_{i}$ are available at present, we estimated $\gamma_{i}$ to be $2.0 \mathrm{erg} \mathrm{cm}^{-2}$ from the theory of Helfand and Tagami ${ }^{24,25}$ and the available data for the solubility parameter and density. ${ }^{18}$ When the entropy term in $\gamma_{i}$ was neglected, the enthalpy change $\left(=\gamma_{\mathrm{i}} s\right)$ due to the change in contact area was found to be 0.002 to $0.03 \mathrm{~J} \mathrm{~cm}^{-3}$. In contrast to the value of $\Gamma s$, these values are 10 to $10^{2}$ times smaller than $\Delta H_{\mathrm{R}}$, indicating that $\Delta H_{\mathrm{R}}$ originates partly from the contact energy $\left(\gamma_{\mathrm{i}} s\right)$ and partly from scission of the main chains or from some other mechanisms. Scission of only ca. $0.01 \%$ of total chains suffices to explain the magnitude of $\Delta H_{\mathrm{R}}$.

Two other mechanisms were considered by Kelterborn and Soong ${ }^{6}$ for the structural breakdown of SBS. In one, polystyrene chains are pulled out of the domains and are mixed with polybutadiene. In the other, voids and crazes are formed in the polystyrene domains. ${ }^{10}$ If it is assumed that about $3 \%$ of polystyrene chains are transferred into the polybutadiene matrix, the magnitude of $\Delta H_{\mathrm{R}}$ can be explained. However, it is difficult to estimate the change in enthalpy due to the second mechanism, since no quantitative data on the sizes of voids and crazes are available at present.

Acknowledgements. The microcalorimeter used in this study was constructed by a support from the Ministry of Education, Science, and Culture (Monbusho) through a Grant 255344. This work was also supported in part by Monbusho under a Grant 543026. They are gratefully acknowledged.

\section{REFERENCES}

1. M. Shen and H. Kawai, Am. Inst. Chem. Eng. J., 24, 1 (1978).

2. T. Hashimoto, M. Fujimura, K. Saijo, H. Kawai, J. Diamant, and M. Shen, "Multiphase Polymers," S. 
L. Cooper and G. M. Estes Eds., American Chemical Society, Washington D.C., 1975, p 257.

3. T. Kotaka, T. Miki, and K. Arai, J. Macromol. Sci., Phys., B17, 303 (1980).

4. H. Kawai, T. Hashimoto, K. Miyoshi, H. Uno, and M. Fujimura, J. Macromol. Sci., Phys., B17, 427 (1980).

5. H. Kawai, T. Hashimoto, K. Miyoshi, H. Uno, and M. Fujimura, Rubber Chem. Technol., 54, 1011 (1981).

6. J. C. Kelterborn and D. S. Soong, Polym. Eng. Sci., 22, 654 (1982).

7. R. M. Yang and E. A. Meinecke, Rubber Chem. Technol., 53, 1124 (1980).

8. J. L. Leblanc, J. Appl. Polym. Sci., 21, 2419 (1977).

9. S. L. Aggarwal, The electron micrographs are shown in ref. 5 .

10. D. H. Kaelble and E. H. Cirlin, J. Polym. Sci., Polym. Symp., No. 43, 131 (1973).

11. C. Price, K. A. Evans, and F. de Candia, Polymer, 14, 338 (1973).

12. C. Price, G. Allen, and N. Yoshimura, Polymer, 16, 261 (1975).
13. G. Allen, C. Price, and N. Yoshimura, Trans. Faraday Soc., 71, 548 (1975).

14. Y. K. Godovsky, Polymer, 22, 75 (1981).

15. L. Araimo, F. de Candia, and V. Vittoria, Polymer, 17, 87 (1976).

16. K. Adachi and T. Kotaka, Polym. J., 14, 959 (1982).

17. E. Guth, Rubber Chem. Technol., 18, 596 (1945).

18. "Polymer Handbook," J. Blandrup and E. H. Immergut, Ed., John Wiley, New York, N. Y., 1975.

19. J. E. Mark, J. Polym. Sci. Macromol. Rev., 11, 135 (1976).

20. R. H. Becher, C. U. Yu, and J. E. Mark, Polym. J., 7, 234 (1975).

21. A. N. Gent and T. H. Kuan, J. Polym. Sci., Polym. Phys. Ed., 11, 1723 (1973).

22. F. de Candia, L. Amelino, and C. Price, J. Polym. Sci., A-2, 10, 975 (1972).

23. J. P. Berry, J. Polym. Sci., 50, 313 (1961).

24. E. Helfand and Y. Tagami, J. Polym. Sci., B, 9, 741 (1971).

25. E. Helfand and A. M. Sapse, J. Chem. Phys., 62, 1327 (1975). 